\title{
The blood coagulation tests from ischemic stroke patients with or without type 2 diabetes mellitus.
}

\author{
Olha Kravchenko $^{1 *}$, Volodymyr Melnyk ${ }^{2}$, Tetiana Tsarenko ${ }^{1}$, Oleksandra Kostiuk ${ }^{1}$, Tetyana Halenova ${ }^{1}$, \\ Nataliia Raksha ${ }^{1}$, Tetyana Vovk ${ }^{1}$, Olexiy Savchuk ${ }^{1}$, Liudmyla Ostapchenko ${ }^{1}$ \\ ${ }^{1}$ Department of Biochemistry, Educational and Scientific Centre, Institute of Biology and Medicine, Taras Shevchenko \\ National University of Kyiv, Kyiv, Ukraine \\ ${ }^{2}$ Department of Neurology, Bogomolets National Medical University, Kyiv, Ukraine
}

\begin{abstract}
Objective: We determined the plasma protein $\mathrm{C}$ and coagulation factor $\mathrm{X}$ activities, prothrombin, fibrinogen and soluble fibrin monomer complex (SFMC) content and performed the haemostatic screening coagulation time tests under acute ischemic stroke patients with or without type 2 diabetes mellitus, as well as evaluated the significance of biochemical haemostatic markers as predictors of mortality in stroke regardless of diabetes presence.

Methods: The baseline data were collected from 87 patients during the admission. Neurological disturbances were assessed using the NIH stroke scale. The functional outcome was estimated using Barthel index. All patients underwent fibrinogen and SFMC (gravimetric methods), prothrombin level (ELISA), plasma protein $\mathrm{C}$ and coagulation factor $\mathrm{X}$ activity assessment, haemostatic screening coagulation time tests with coagulation analyzer, glucose and glycosylated hemoglobin content and BMI (body mass index) measurements.

Results: The conducted research had established the changes of fibrinogen and SFMC levels in both investigated patient groups comparing to the control. The protein $\mathrm{C}$ activity was found to be significantly decreased in blood of patients with ischemic stroke with and without diabetes. There were differences in factor $\mathbf{X}$ activity change in patients with stroke only comparing with patients with diabetes and stroke and high level of this parameter as well as the increase in SFMC can be regarded as death predictors of stroke independently of diabetes mellitus presence.

Conclusion: Among ischemic stroke patients with type 2 diabetes mellitus the differences were more significant for all Time tests of the coagulation cascade, but deviations of haemostatic biochemical markers were more pronounced in ischemic stroke patients without diabetes mellitus.
\end{abstract}

Keywords: Stroke, Diabetes mellitus, Type 2, Blood coagulation tests, Blood coagulation factors. Accepted on July 02, 2018

\section{Introduction}

The haemostatic system has several important functions such as maintaining blood in a fluid state, arresting bleeding at the site of vascular injury or blood loss by forming a haemostatic plug, and ensuring the eventual removal of the clot when healing takes place. Normal physiology constitutes a delicate balance between pro-coagulant and anticoagulant properties of this system, and deficiency or exaggeration of any of its components may lead to either hemorrhage or vascular thrombosis, respectively [1]. Exactly the latter disturbance in blood vessels inside the brain appears to be the cause of ischemic thrombotic stroke. This type of stroke is usually seen among elderly people, especially those with atherosclerosis, hypertension and diabetes mellitus (DM).
One of the most important plasma proteins which are critical for hemostasis and clot formation is fibrinogen. Its cleavage by thrombin (coagulation factor II) and subsequent polymerization forming fibrin strands provides the structural network required for effective clot formation [2]. Besides a complex coupling of fibrinogen molecules and fibrin monomers named the soluble fibrin monomer complex (SFMC) is formed in the earlyactivated state of blood coagulation. Thus such molecular complex appearance is expected to serve as a marker for the diagnosis of thrombotic formation and disseminated intravascular coagulation, in particular at its early stage [3]. Soluble fibrin complexes accumulation in blood plasma is accompanied by proportional coagulation time reduction in ancistron time tests (AT, reptilase time). Other haemostatic screening coagulation time tests, particularly evaluating the level and function of fibrinogen are thrombin time (TT), 
prothrombin time $(\mathrm{PT})$, activated partial thromboplastin time (APTT) show the speed of blood clot formation [4].

Similar to blood coagulation factors II (prothrombin), which after activation converts fibrinogen, factor $\mathrm{X}$ and the protein $\mathrm{C}$ synthesis in the liver depends on the presence of vitamin $\mathrm{K}$. However, in contrast to the other coagulation factors, protein $\mathrm{C}$, when activated by thrombin, is not a pro-coagulant but a potent anticoagulant.

Abnormalities in the plasmatic coagulation system have been repeatedly described in patients with DM [5] and have been linked to a variety of vascular diseases, including atherosclerosis and ischemic stroke (IS), which have been associated with hemostasis disorders as well [6]. Besides fibrinogen level at admission there is a factor that might play a significant role in the efficacy and mortality associated with thrombolytic therapy in patients with stroke $[7,8]$ and increase mortality in remote periods [9].

So the aim of the present study was to determine the plasma protein $\mathrm{C}$ (PC), coagulation factor $\mathrm{X}$ activities, prothrombin, fibrinogen and SFMC content and haemostatic screening coagulation time tests (TT, PT, APTT and AT) under acute ischemic stroke (IS) in patients with or without type 2 diabetes mellitus and evaluate the significance of biochemical haemostatic markers as predictors of mortality in stroke regardless of diabetes.

\section{Material and Methods}

The case-control study included 87 patients (median age, 74.2 \pm 9.0 y) who were admitted due IS to Kyiv City Hospital \#4 and 25 population controls (median age, $70.2 \pm 10.3 \mathrm{y}$ ).

The diagnosis of IS was confirmed by clinical symptoms and brain CT and MRI (T1, T2 and DWI) in 87 patients, 23 (26.4\%) of which had type 2 DM diagnosed according to the 1998 World Health Organization guidelines [10].

The baseline data were collected for all patients during the admission, including sex, age and comorbidities. Neurological disturbances were assessed at the admission (day 1), at day 7 and day 14 using the NIH Stroke Scale (NIHSS). The functional outcome was assessed at 7 and 14 day using Barthel index.

All the patients underwent fibrinogen and SFMC, prothrombin level, plasma protein $\mathrm{C}$ (PC) and coagulation factor $\mathrm{X}$ activity assessment, TT, PT, APTT, AT, glucose and glycosylated hemoglobin content and BMI (body mass index) measurements.

Patients were excluded if they had a personal history of predisposition to hypercoagulability, coma, cancer, recent surgery, and hyperthyroidism or liver pathologies.

This study was approved by the ethic committee of the Educational and Scientific Centre "Institute of Biology" of Taras Shevchenko National University of Kyiv, Ukraine, and was in accordance with the Helsinki declaration. Written informed consent was obtained from each of the participants at the time of enrolment.

All the patients had management of ischemic stroke according to Guidelines for Management of Ischaemic Stroke and Transient Ischaemic Attack ESO, 2008 and aspirin therapy in dose $325 \mathrm{mg}$ during the first $24 \mathrm{~h}$ after first symptoms appeared $(6.2 \pm 2.7 \mathrm{~h})$. The blood for hemostasis investigations was collected from the patients shortly after their admission to the hospital (acute phase of stroke) in tubes containing 1/10 volume of $0.129 \mathrm{~mol} / \mathrm{L}$ sodium citrate. For DM identifying venous fasting blood was collected from the cubital vein from 8 to 9 am in a test tube with the same solution of sodium citrate. The glucose content was estimated by enzymatic glucose oxidase method.

Fibrinogen plasma content was estimated by gravimetry with following spectrophotometry assays [11]. Isolation of SFMC was done using the phosphate method with $0.1 \mathrm{M}$ phosphatebuffered saline containing sodium citrate and $0.2 \%$ 6aminohexanoic acid with following determination [12].

\section{Prothrombin content was determined by ELISA}

All other indexes (protein $\mathrm{C}$ and coagulation factor $\mathrm{X}$ activities, TT, PT, APTT, AT) were measured with the coagulation method on a Rayto RT-2201C Coagulation Analyzer (Rayto, Shenzhen, China) using Renam reagents (Moscow, Russia). Glycosylated haemoglobin was determined on a spectrophotometer Bio-Rad with HbA1 IMU-LA-TEST (Erba Lachema S.R.O. Czech Republic). BMI as an index to estimate the extent of obesity was calculated as body weight $(\mathrm{kg})$ divided by the square of the height $\left(\mathrm{m}^{2}\right)$.

The data entry and analysis were performed using Statsoft Statistica version 7.0 for Windows. Means and standard deviations were calculated for numerical variables. The significance of differences of prothrombin, fibrinogen and SFMC, protein $\mathrm{C}$ and coagulation factor $\mathrm{X}$ activities, glucose content, age and BMI between groups were determined using the Mann-Whitney U test.

TT, PT, APTT, AT data was heterogeneous and abnormally distributed so they were subdivided into shortened, control and lengthened groups. Frequencies and percentages were calculated for all categorical variables. The significance of differences in time tests of the coagulation cascade between the groups was compared with the Fisher exact test for TT and PT, Pearson's Chi-square test for APTT and AT.

All statistical tests were 2 -tailed with $\mathrm{p}$-values $<0.05$ taken as significant.

\section{Results and Discussion}

All 87 patients included to the investigation were analyzed. Their main clinical characteristics are presented in Table 1. There were no significant differences between patients with or 
without DM in sex, age, hypertension and ischemic heart disease presence.

Table 1. The main clinical characteristics of patients with ischemic stroke (IS) and patients with ischemic stroke and type 2 diabetes (IS $+D M)$.

\begin{tabular}{llll}
\hline & IS $(\mathbf{n}=64)$ & IS+DM $(\mathbf{n}=\mathbf{2 3})$ & $\mathbf{p}$ \\
\hline Age, $y(\mathrm{M} \pm \mathrm{SD})$ & $76.2 \pm 10.9$ & $73.8 \pm 9.7$ & 0.503 \\
\hline Sex, male, $\mathrm{n}(\%)$ & $32(50)$ & $8(34.9)$ & 0.214 \\
\hline Hypertension, $\mathrm{n}(\%)$ & $45(70.3)$ & $17(73.9)$ & 0.537 \\
\hline Hyperlipidaemia, $\mathrm{n}(\%)$ & $20(31.2)$ & $11(47.8)$ & $<0.001$ \\
\hline Ischemic heart disease, $\mathrm{n}(\%)$ & $19(29.7)$ & $8(34.9)$ & 0.120 \\
\hline Peripheral artery disease, $\mathrm{n}(\%)$ & $6(9.4)$ & $4(17.3)$ & $<0.001$ \\
\hline Body mass index, $\mathrm{kg} / \mathrm{m}^{2}$ & $20.26 \pm 1.6$ & $34.01 \pm 5.48$ & 0.023 \\
\hline Baseline NIHSS $(\mathrm{M} \pm \mathrm{SE})$ & $9.9 \pm 0.5$ & $12.2 \pm 1.0$ & 0.030 \\
\hline Barthel index at $7 \mathrm{~d}(\mathrm{M} \pm \mathrm{SE})$ & $66.4 \pm 2.6$ & $57.3 \pm 5.9$ & 0.117 \\
\hline
\end{tabular}

Dyslipidemia and body mass index (BMI) assessments revealed significantly high indexes in case of type 2 diabetes with ischemic stroke; the results showed IS patients had BMI within the normal range $-20.26 \pm 1.6 \mathrm{~kg} / \mathrm{m}^{2}$, while under ischemic stroke with diabetes, this value was $34.01 \pm 5.48$ $\mathrm{kg} / \mathrm{m}^{2}, \mathrm{p}=0.023$. The baselines of NIHSS $(\mathrm{p}=0.030)$ were lower in IS patients comparing with the results of IS+DM patients. Dynamics of neurological disturbances of ischemic stroke patients with or without DM were as follows. The initially significantly deeper neurological deficits $(12.2 \pm 1.0,95 \% \mathrm{CI}$ 10.3-14.0) were observed in the IS patients with DM if compared with the IS patients without DM $(9.9 \pm 0.89,95 \% \mathrm{CI}$ $8.9-10.9, p=0.030$ ). This difference was still evident at the $7^{\text {th }}$ and $14^{\text {th }} \mathrm{d}$ of the disease in spite of the general tendency to reduce the negative manifestations. The established differences of the neurological deficits severity were confirmed by the expected differences in the outcome of functional Barthel Index (Day 7 under IS-66.4 \pm 2.6 95\% CI 61.2-71.5 and under IS+DM-57.3 $\pm 5.995 \%$ CI 44.8-69.9, $\mathrm{p}=0.117$; Day 14 under IS-75.1 $\pm 2.595 \%$ CI $69.9-80.2$ and under IS+DM-69.4 \pm 5.2 $95 \%$ CI 58.4-80.4, $\mathrm{p}=0.297$ ).

Despite the on-going intensive therapy of ischemic stroke 15 $(17.2 \%)$ patients died within the first two weeks (average $5.3 \pm$ 1.9 d) among which were $10(20.8 \%)$ females and $5(12.8 \%)$ males. The patients with IS+DM had an excess mortality over IS patients without DM during the first $14 \mathrm{~d}$ after apoplexy which caused hospitalization. So 9 (14.1\%) cases of IS had lethal outcomes and IS with diabetes had lethality in 6 cases ( $26.1 \%$ from all patients with this pathologies, $p=0.161$ ).

The group of patients with IS+DM included individuals whose mean blood glucose content was $9.47 \pm 2.65 \mathrm{mmol} / \mathrm{L}$, while in the group with IS this parameter was $4.93 \pm 0.97 \mathrm{mmol} / \mathrm{L}$.

The conducted research has established the statistically significant changes of fibrinogen and SFMC levels in both investigated patient groups in comparison with the control
(Figure 1). Namely the fibrinogen blood content reached 130\% and $135 \%$ in IS and IS+DM group respectively if compared with the control.

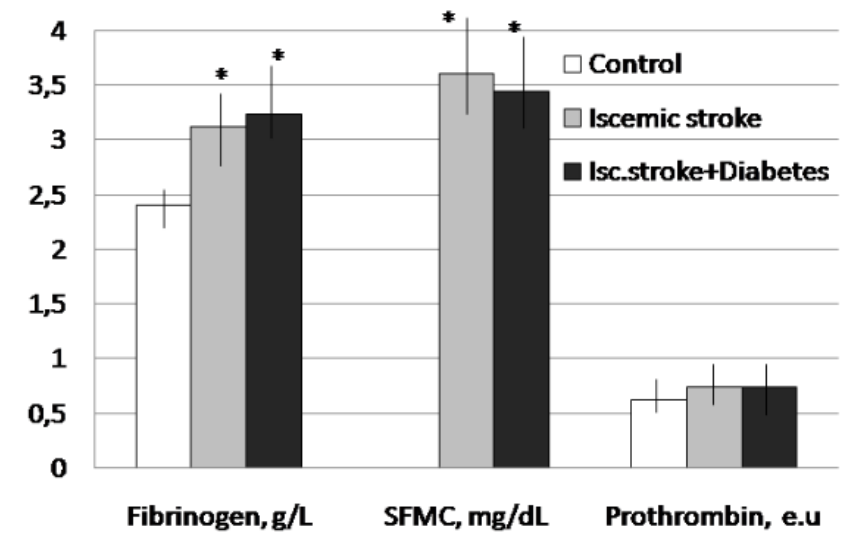

Figure 1. Fibrinogen, soluble fibrin-monomer complexes (SFMC), prothrombin plasma content under ischemic stroke and ischemic stroke in the patients with type 2 diabetes. Mean \pm Standard Deviation $(S D) .{ }^{*} P$ value is significant when $\leq 0.05$ in comparison with the control.

SFMC was not detected in apparently healthy donors' blood but in the case of the investigated pathologies the quantity of these soluble fibrin monomer/fibrinogen complexes was significantly higher.

It should be noted that fibrinogen indexes were higher in the case of IS with DM, whereas SFMC had more deviated meanings if compared with the control parameters under ischemic stroke only (Figure 1). Nevertheless statistically significant differences of fibrinogen and SFMC levels in the patients with ischemic stroke and stroke complicated by diabetes were not detected.

The established index of prothrombin content was $0.74 \pm 0.18$ e.u. in blood of IS+DM patients and $0.74 \pm 0.199$ e.u. in blood of patients with IS only. The benchmark of prothrombin content for apparently healthy donors' blood was $0.63 \pm 0.15$ e.u (Figure 1).

Thus the plasma prothrombin content was increased by $18 \%$ from the control for both researched groups of patients and no significant differences of prothrombin amount were detected in the patients with both researched diseases and these parameters were non-significantly higher if compared with the control.

The protein $\mathrm{C}$ (PC) activity was found to be significantly decreased in blood of the patients with IS like IS+DM subjects if compared with the controls (Figure 2). The estimated reduction of PC activity was more evident in the case of IS only.

The plasma coagulation factor $\mathrm{X}$ activity was increased only under IS accounting for $117 \%$ of the controls. There were detected differences in changes of factor $\mathrm{X}$ activity from patients with stroke only comparing to patients with diabetes and stroke. 


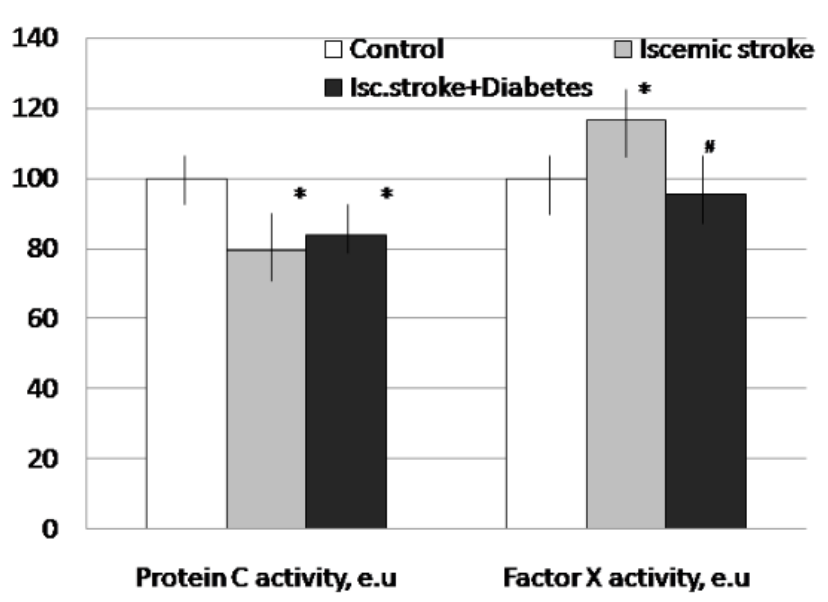

Figure 2. Protein $C$ and Factor X plasma activities under is-chemic stroke and ischemic stroke in the patients with type 2 diabetes. Mean \pm Standard Deviation (SD). ${ }^{*} P$ value is significant when $\leq 0.05$ in comparison with the control. ${ }^{\# P}$ value is significant when $\leq 0.05$ in comparison with the Ischemic stroke.

There were analyzed the indexes of fibrinogen, concentrations of SFMC and factor $\mathrm{X}$ activity and patients survival for identifying death predictors of stroke independently of diabetes mellitus presence. The patients who survived during $14 \mathrm{~d}$ after admission to hospital were characterized significantly lower concentration of SFMC $(3.31 \pm 1.4 \mathrm{mg} / 100 \mathrm{ml} \mathrm{95 \%} \mathrm{CI}$ 3.09-3.67) compared with patients who died $(3.83 \pm 1.5$ $\mathrm{mg} / 100 \mathrm{ml} 95 \%$ CI 3.57-4.29; $\mathrm{p}=0.033)$. The patients who died, on admission to hospital showed significantly higher $\mathrm{X}$ factor activity compared with survived patients $(105.13 \pm 31.0$ and $88.13 \pm 30.1 \%$ respectively, $\mathrm{p}=0.049$ ) (Table 2 ).

Table 2. Predictors of mortality under acute ischemic stroke.

\begin{tabular}{llllllll}
\hline & & M & m & SE & $95 \%$ Cl & p \\
\hline Fibrinogen, g/L & Survived & 3.07 & 0.74 & 0.08 & 2.89 & 3.25 & 0.552 \\
\hline
\end{tabular}

\begin{tabular}{|c|c|c|c|c|c|c|c|}
\hline & Dead & 3.15 & 0.77 & 0.20 & 2.52 & 3.38 & \\
\hline \multirow{2}{*}{ SFMC, $\mathrm{mg} / 100 \mathrm{ml}$} & Survived & 3.31 & 1.36 & 0.16 & 3.09 & 3.67 & \multirow{2}{*}{$0.033^{\dagger}$} \\
\hline & Dead & 3.83 & 1.55 & 0.40 & 3.57 & 4.29 & \\
\hline \multirow{2}{*}{$\begin{array}{l}\text { Factor X activity, } \\
\%\end{array}$} & Survived & 88.13 & 30.15 & 7.79 & 72.83 & 106.23 & \multirow{2}{*}{$0.049^{\dagger}$} \\
\hline & Dead & 105.13 & 31.03 & 3.66 & 97.83 & 112.42 & \\
\hline
\end{tabular}

tThe result is significant at $p<0.05$.

Thus the high blood levels of SFMC and factor X activity in the patients with ischemic stroke on admission to a hospital provided the basis for negative survival forecast of such patients. Thus these indicators influence the rate and efficiency of blood clotting, the time tests of the coagulation were investigated (Table 3 ). The trend towards lengthening of all the investigated chronometric parameters hemostasis was revealed.

$31(48.5 \%)$ patient showed lengthened TT under ischemic stroke and $33(51.5 \%)$ patients had normal TT. This parameter (diabetes complicated by stroke) had more severe changes, so only $4(17.5 \%)$ from 23 patients with IS+DM had normal TT; others were characterized by prolonged thrombin time. TT was lengthened significantly in the IS+DM patients compared with the IS group ( $p$ value 0.00623 ) as shown in Table 3. The maximum value of the thrombin time registered for patients with ischemic stroke was $29 \mathrm{~s}$ and $27.4 \mathrm{~s}$ for stroke complicated by diabetes.

Other time coagulation tests showed increase of blood clotting indexes under the investigated pathologies. In addition more expressed rise was observed in case of glycaemia presence. The quantity of patients with ischemic stroke who had lengthened PT and APTT amounted to $45.5 \%$ and $40.5 \%$ respectively, on the other hand the number of diabetes with IS patients with such abnormal parameters was about $60 \%$ ( 56.5\%-for PT, 61\%-for APTT).

Table 3. Time tests of the coagulation cascade in blood of patients with ischemic stroke and stroke with diabetes mellitus.

\begin{tabular}{|c|c|c|c|c|}
\hline \multicolumn{4}{|l|}{ Thrombin time (TT) } & \multirow[t]{2}{*}{$\mathbf{p}$} \\
\hline & $<10 \mathrm{~s}$ & Control,10-16.5 s & $>16.5 \mathrm{~s}$ & \\
\hline Ischemic stroke & 0 & $33(51.5 \%)$ & $31(48.5 \%)$ & \multirow{2}{*}{$0.00623^{\dagger}$} \\
\hline Iscemicstroke+Diabetes mellitus & 0 & $4(17.5 \%)$ & $19(82.5 \%)$ & \\
\hline \multicolumn{5}{|l|}{ Prothrombin time (PT) } \\
\hline & $<13 \mathrm{~s}$ & Control, $13-19 \mathrm{~s}$ & $>19 \mathrm{~s}$ & \\
\hline Ischemic stroke & 0 & $35(54.5 \%)$ & $29(45.5 \%)$ & \multirow{2}{*}{0.466535} \\
\hline Iscemic stroke+Diabetes mellitus & 0 & $10(43.5 \%)$ & $13(56.5 \%)$ & \\
\hline \multicolumn{5}{|c|}{ Activated partial thromboplastin time (APTT) } \\
\hline & $<25 \mathrm{~s}$ & Control, $25-45 \mathrm{~s}$ & $>45 \mathrm{~s}$ & \\
\hline Ischemic stroke & $1(1.5 \%)$ & $37(58 \%)$ & $26(40.5 \%)$ & \multirow{2}{*}{0.224871} \\
\hline Iscemic stroke+Diabetes mellitus & 0 & $9(39 \%)$ & $14(61 \%)$ & \\
\hline
\end{tabular}




\begin{tabular}{llll}
\hline Ancistron (Reptilase) time & & & \\
\hline & $<16 \mathrm{~s}$ & Control, 16-20 s & $>20 \mathrm{~s}$ \\
\hline Ischemic stroke & $18(28 \%)$ & $41(64 \%)$ & $5(8 \%)$ \\
\hline Iscemic stroke+Diabetes mellitus & $3(13 \%)$ & $17(74 \%)$ & $3(13 \%)$ \\
\hline
\end{tabular}

tThe result is significant at $p<0.05$.

Ancistron time tests, characterizing clot formation independently from the presence of unfractionated and low molecular weight heparin, direct thrombin inhibitors and warfarin in blood stream, were normal for $64 \%$ IS patients and $74 \%$ IS+DM patients. There were patients who had shortened Ancistron time, including 28\% among IS patients and $13 \%$ in case of ischemic stroke with diabetes. The same quantity (13\%) from the DM cohort had lengthened Ancistron time and only $8 \%$ persons with IS had this parameter increased. It should be noted that, we haven't identified statistically significant differences of PT, APTT and Ancistron time among ischemic stroke patients with or without type 2 diabetes mellitus.

Lots of evidences have demonstrated fibrinogen to be a powerful risk marker for cardio- and cerebrovascular diseases in the general population, and it is also likely to contribute toward the increased atherosclerotic risk in DM, especially in type $2 \mathrm{DM}$. The aetiology of hyperfibrinogenemia in DM is likely to be multifactorial, and at present the mechanisms involved have not been clarified [13]. Besides, an elevated serum fibrinogen value is common following ischemic stroke, thus Fibrinogen like Fibrin degradation products, protein $\mathrm{C}$, Factor II and X, TT etc. are included to coagulation tests for evaluation of patients with transient ischemic attack or ischemic stroke [14]. The conducted research has established the statistically significant changes of fibrinogen and soluble fibrin monomer complexes (SFMC) levels under both investigated pathologies (ischemic stroke and ischemic stroke complicated by insulin-independent diabetes mellitus). SFMC, the early marker of thrombophilia, was significantly higher compared with the reference values in case of stroke as well as stroke with diabetes. It was established that fibrinogen indexes were higher under IS with DM, whereas SFMC had more deviated meanings comparing with the control parameters under ischemic stroke only. The higher SFMC level in acute phase of stroke independently of diabetes presence appears to be a statistically confirmed predictor of mortality.

Nevertheless statistically significant differences of fibrinogen and SFMC levels in patients with ischemic stroke and IS+DM were not detected and there were no differences in protrombin content neither if compared with the control nor between the studied pathologies. The only parameter from all researched by us having tendency to decrease was anticoagulant protein $\mathrm{C}$ activity. As the levels of coagulation factor II and factor $\mathrm{X}$ activity were not reduced neither in the ischemic stroke patients nor in the IS+DM patients, the reduction of protein $\mathrm{C}$ seems to be caused not because of its synthesis diminishment in the liver or vitamin $\mathrm{K}$ deficiency, but more likely due to its increased clearance speed from the blood plasma. It should be noted that only factor $\mathrm{X}$ activity had significantly different changes in IS and IS+DM patients. The blood from patients with ischemic stroke was characterized by the increase of the aforementioned index and this parameter had tendency to be reduced in the hyperglycemia presence.

The defining precursors of fatal stroke outcomes independently of diabetes mellitus presence, revealed the decreased factor $\mathrm{X}$ activity at the hospital admission time as a positive predictor for survival of patients.

The presence of circulating soluble fibrin monomer complexes in plasma, hyperfibrinogenemia, the decrease of protein $\mathrm{C}$ activity accompanied by the increase of factor $\mathrm{X}$ activity with high protormbin content in the plasma of patients with ischemic stroke and type 2 diabetes with stroke patients indicates abnormal, probably hypercoagulable conditions in aforementioned disorders.

Despite the increase in procoagulation components in the blood stream of the patients with IS and IS+DM, the increase of the number of the patients with almost all time tests of the coagulation cascade lengthened was established. These results are consistent with the data by Margaret Todd and may be due to vitamin $\mathrm{K}$ metabolism disturbances (vitamin $\mathrm{K}$ antagonist therapy), failure of blood clotting factors functioning-for PT and APTT and the presence of coagulation cascade nonphysiological inhibitors, appearing in blood stream as a result of diseases or even treatment with heparin or other anticoagulants. It has been suggested by Fletcher that there is a fundamental abnormality in fibrin polymerization in patients with complications of atherosclerotic vascular disease $[15,16]$, but this assumption is not supported by relatively normal rates of Ancistron (Reptilase) time registered in both researched pathologies.

\section{Conclusion}

The obtained results have shown that ischemic stroke with and without type 2 diabetes mellitus both are characterized by similar changes of the investigated hemostasis parameters, excluding Factor $\mathrm{X}$ activity. The higher quantity of blood SFMC, and the presence of more active factor $\mathrm{X}$ simultaneously with the depletion of anticoagulant protein $\mathrm{C}$ activity were established in the patients' blood with stroke only. The differences in all time tests of the coagulation cascade were more significant among the ischemic stroke patients with diabetes mellitus. Although the ischemic stroke 
was characterized by a greater percentage of patients with lengthened PT, APTT and TT if compared with the control, the presence of DM was able to reinforce the negative manifestations of the changes in coagulation test parameters.

\section{Acknowledgment and Sources of Funding}

The National Government of Ukraine.

\section{Conflict of Interest}

None.

\section{References}

1. Abdullah WZ, Moufak SK, Yusof Z, Mohamad MS Kamarul IM. Shortened activated partial thromboplastin time, a hemostatic marker for hypercoagulable state during acute coronary event. Translational Res: J Laboratory Clin Med 2010; 155: 315-319.

2. Jerrold H. Levy, FaniaSzlam, Kenichi A. Tanaka, Roman M. Sniecienski. Fibrinogen and hemostasis: A primary hemostatic target for the management of acquired bleeding. Anesth Analgesia 2012; 114: 261-274.

3. Koga S. A novel molecular marker for thrombus formation and life prognosis--clinical usefulness of measurement of soluble fibrin monomer-fibrinogen complex (SF). RinshoByori 2004; 52: 355-361.

4. David JP, Tony T. A practical guide to laboratory haemostasis. Practical-Haemostasis 2016.

5. Fayeza K, Qazi SA, Shamima J, Afruza K, Samira H, Tania Y. Coagulation impairment in type 2 diabetes mellitus. J Bangladesh Soc Physiol 2015; 10: 26-29.

6. Rothwell PM, Howard SC, Power DA, Gutnikov SA, Ale Algra, Jan van G. Fibrinogen concentration and risk of ischemic stroke and acute coronary events in 5113 patients with transient ischemic attack and minor ischemic stroke. Stroke 2004; 35: 2300-2305.

7. Swarowska M, Polczak A, Pera J, Klimkowicz-Mrowiec A, Slowik A, Dziedzic T. Hyperfibrinogenemia predicts long-term risk of death after ischemic stroke. J Thromb Thrombolysis 2014; 38: 517-521.

8. Gonzalez-Conejero R, Fernandez-Cadenas I, Iniesta JA, Marti-Fabregas J, Obach V, Alvarez-Sabin J. Role of fibrinogen levels and factor XIII V34L polymorphism in thrombolytic therapy in stroke patients. Stroke 2006; 37 : 2288-2293.

9. Turaj W, Slowik A, Dziedzic T, Pulyk R, Adamski M, Strojny J. Increased plasma fibrinogen predicts one-year mortality in patients with acute ischemic stroke. J Neurol Sci 2006; 246: 13-19.

10. Alberti KG, Zimmet PZ. Definition, diagnosis and classification of diabetes mellitus and its complications. Part 1: diagnosis and classification of diabetes mellitus. Provisional report of a WHO consultation. Diabet Med 1998; 15: 539-553.

11. Tokar AV, Makogonenko EM, Platonova TM. Modern methods of intravenous micro-coagulation laboratory diagnostic (guidelines). Maccom, Kyiv 1994.

12. Zaichko NV, Chernyshenko TM, Platonova TM, Volkov GL. Influence of soluble fibrin on the blood coagulation process and platelets aggregation. The Ukr Biochem J 2006; 78: 118-123.

13. Dunn EJ, Ariëns RA. Fibrinogen and fibrin clot structure in diabetes. Herz 2004; 29: 470-479.

14. Harold PA, Vladimir $\mathrm{CH}$, John WN. Ischemic cerebrovascular disease. Oxford University Press, New York 2001.

15. Margaret T, Ellen M, Fletcher M. Stroke and blood coagulation. Stroke 1973; 4: 400-405.

16. European Stroke Organization. Guidelines for management of ischaemic stroke and transient ischaemic attack. Cerebrovasc Dis 2008; 25: 457-507.

\section{*Correspondence to}

Olha Kravchenko

Department of Biochemistry

Educational and Scientific Centre

Institute of Biology and Medicine

Taras Shevchenko National University of Kyiv

Kyiv

Ukraine 